\title{
Sorption and transformation of the reactive tracers resazurin and resorufin in natural river sediments
}

\author{
D. Lemke ${ }^{1,2}$, R. González-Pinzón ${ }^{3}$, Z. Liao ${ }^{1}$, T. Wöhling ${ }^{2}$, K. Osenbrück ${ }^{2}$, R. Haggerty ${ }^{4}$, and O. A. Cirpka ${ }^{1}$ \\ ${ }^{1}$ University of Tübingen, Center for Applied Geoscience, Hölderlinstr. 12, 72074 Tübingen, Germany \\ ${ }^{2}$ Water and Earth System Science (WESS) Competence Cluster, University of Tübingen, \\ Hölderlinstr. 12, 72074 Tübingen, Germany \\ ${ }^{3}$ University of New Mexico, Department of Civil Engineering, 210 University Blvd NE, Albuquerque, NM 87131, USA \\ ${ }^{4}$ Oregon State University, College of Earth, Ocean and Atmospheric Sciences, 104 Wilkinson Hall, \\ Corvallis, OR 97331-5506, USA
}

Correspondence to: O. A. Cirpka (olaf.cirpka@uni-tuebingen.de)

Received: 18 July 2013 - Published in Hydrol. Earth Syst. Sci. Discuss.: 8 October 2013

Revised: 28 April 2014 - Accepted: 17 July 2014 - Published: 25 August 2014

\begin{abstract}
Resazurin (Raz) and its reaction product resorufin (Rru) have increasingly been used as reactive tracers to quantify metabolic activity and hyporheic exchange in streams. Previous work has indicated that these compounds undergo sorption in stream sediments. We present laboratory experiments on Raz and Rru transport, sorption, and transformation, consisting of 4 column and 72 batch tests using 2 sediments with different physicochemical properties under neutral $(\mathrm{pH}=7)$ and alkaline $(\mathrm{pH}=9)$ conditions. The study aimed at identifying the key processes of reactive transport of Raz and Rru in streambed sediments and the experimental setup best suited for their determination. Data from column experiments were simulated by a travel-timebased model accounting for physical transport, equilibrium and kinetic sorption, and three first-order reactions. We derived the travel-time distributions directly from the breakthrough curve (BTC) of the conservative tracer, fluorescein, rather than from fitting an advective-dispersive transport model, and inferred from those distributions the transfer functions of Raz and Rru, which provided conclusive approximations of the measured BTCs. The most likely reactive transport parameters and their uncertainty were determined by a Markov chain-Monte Carlo approach. Sorption isotherms of both compounds were obtained from batch experiments. We found that kinetic sorption dominates sorption of both Raz and Rru, with characteristic timescales of sorption in the order of 12 to $298 \mathrm{~min}$. Linear sorption models for both Raz and Rru appeared adequate for concentrations that
\end{abstract}

are typically applied in field tracer tests. The proposed twosite sorption model helps to interpret transient tracer tests using the Raz-Rru system.

\section{Introduction}

Resazurin (Raz) undergoes irreversible reduction to resorufin (Rru) in the presence of cellular metabolic activity. Lab experiments (Haggerty et al., 2008; Stanaway et al., 2012) and field tracer tests (Haggerty et al., 2009; González-Pinzón et al., 2012) have shown that the transformation of Raz to Rru is exceptionally favored in the presence of hyporheic sediments; that is, reaction rates in the presence of colonized sediments are typically 3 orders of magnitude larger than in the water column, and strongly correlated to respiration (Haggerty et al., 2008; González-Pinzón et al., 2012). This reaction can be used to estimate metabolic activity in hydrologic systems (Haggerty et al., 2009; Argerich et al., 2011; González-Pinzón et al., 2014). It has also been used to separate effects of in-stream mixing processes from exchange processes with comparably immobile, metabolically active zones adjacent to streams in the interpretation of stream tracer tests (Lemke et al., 2013a; Liao et al., 2013).

In field and column experiments, the breakthrough curves (BTCs) of Raz and Rru may exhibit tailing and may be retarded compared to the BTCs of conservative tracers. Additionally, these experiments have been characterized by an 
incomplete mass balance between the amount of Raz injected and that of Raz and Rru recovered. The latter observation suggests that Raz and Rru are affected by irreversible sorption and/or transformation to undetected metabolites, preventing complete mass recovery within typical experimental timescales. It is widely known that sorption affects the fate and transport of organic compounds by mass retention on various timescales (Miller and Weber, 1988; Weber et al., 1991; Piwoni and Keeley, 1990), so that sorption processes need to be considered in transient mass transport studies.

To date, little work has been done to characterize the sorption of Raz and Rru. Haggerty et al. (2008) fitted linear and non-linear (Freundlich) isotherms to batch experiments on equilibrium sorption of Rru in stream sediments containing $\sim 2 \%$ organic carbon for concentrations of up to $100 \mu \mathrm{g} \mathrm{L}^{-1}$. The distribution coefficient of the tracers between the solid and aqueous phases in equilibrium, denoted $K_{\mathrm{d}}$, derived by the linear sorption isotherm was estimated as $6.63 \mathrm{~L} \mathrm{~kg}^{-1}$, which corresponds to a retardation factor of up to 60 in sediments. This value most likely overestimates the sorption capacity and is presumably attributed to using a disturbed sample and disregarding the transformation of Rru to nondetected metabolites in the batch sample. Quantifying the sorption of Raz onto natural, metabolically active sediments is difficult with conventional batch experiments due to the rapid reaction of Raz to Rru, so that no such studies have been conducted so far.

In previous studies, sorption of Raz and Rru has been modeled inconsistently. Several studies assumed linear equilibrium sorption (Haggerty et al., 2008; Stanaway et al., 2012; Lemke et al., 2013a), whereas Liao et al. (2013) applied a more sophisticated model, considering both equilibrium and kinetic linear sorption. Moreover, some studies assumed identical equilibrium sorption properties of both Raz and Rru (Haggerty et al., 2008, 2009; Stanaway et al., 2012), whereas others allowed the sorption parameters of these two compounds to differ (Lemke et al., 2013a; Liao et al., 2013). The choices of how sorption processes were implemented in the studies cited above were predominantly guided by simplifying assumptions (i.e., that the similar molecular structure of Raz and Rru should lead to approximately identical sorption properties) or the desire to keep the computational effort of the model low. Differing model assumptions add uncertainty to a comparison of the previous results, and the previous models used may be oversimplified regarding sorption processes.

In this paper, we present the results of 72 laboratory batch and 4 column experiments on sorption of Raz and Rru. We have chosen two different sediments with different physicochemical properties and conducted all experiments at two different $\mathrm{pH}$ values $(\mathrm{pH} 7$ and $\mathrm{pH}$ 9) to cover a range of natural conditions under which tracer tests using Raz, Rru, and the conservative tracer fluorescein are considered feasible. By this we aim at identifying (1) the relative importance of kinetic and equilibrium processes for the sorption of Raz and Rru in streambed sediments, (2) the level of model complexity required to adequately reproduce the measured BTCs during field tracer tests, and (3) the experimental setup best suited for determination of the sorption and reaction parameters of Raz and Rru.

Although laboratory experiments may provide important insight into the relevance of different sorption processes, they often fail to reproduce realistic field conditions. Therefore, the determination of single parameter values and their possible interrelations with specific physicochemical conditions or sediment properties is not the primary focus of this study. We rather tried to elucidate the relative importance of the different processes (e.g., kinetic or equilibrium sorption, linear or non-linear isotherms, decay of Raz to Rru or nonfluorescent compounds) that potentially control transport of Raz and Rru in streambed sediments. Due to the expected high intra- as well as inter-stream variability of the transport properties of the reactive tracers, a comprehensive evaluation of sorption parameters and their controlling environmental properties was beyond the scope of this study. Instead, we suggest an experimental framework that might be applied to other sites.

\section{Materials and methods}

\subsection{River sediments}

Sediments were taken from the third-order stream River Goldersbach $\left(48^{\circ} 33.298^{\prime} \mathrm{N}, 9^{\circ} 4.002^{\prime} \mathrm{E}\right)$ and the fourth-order stream River Steinlach $\left(48^{\circ} 28.585^{\prime} \mathrm{N}, 9^{\circ} 3.818^{\prime} \mathrm{E}\right)$, which are located close to Tübingen in the southwestern part of Germany. River Goldersbach has a mean annual discharge of $0.3 \mathrm{~m}^{3} \mathrm{~s}^{-1}$. Its catchment and the river sediments are dominated by sand- and marlstones of the Upper Triassic. River Steinlach has a mean annual discharge of $1.7 \mathrm{~m}^{3} \mathrm{~s}-1$. Its riverbed is mainly composed of limestone originating from the nearby Jurassic Swabian Alb mountains.

The sediments used in the experiments were sieved in the field to grain sizes between 0.08 and $4 \mathrm{~mm}$. Each sediment sample was washed to remove residues of finer particle sizes that increase the turbidity of the tracer solution and thus have a negative influence on the accuracy of the tracer measurements (Lemke et al., 2013b). Minimizing turbidity is critical to assure the required high-quality fluorescence measurements and to assure the necessary comparability of the different experiments. However, discarding the finest sediment fraction might introduce a bias to the derived reaction and sorption parameters as compared to those prevailing in the field, which has to be accounted for in the interpretation of the experiments. The time between the collection of the sediment and the start of the column experiments was less than $5 \mathrm{~h}$ in every case. 
The organic carbon content of the sediments was determined by standard titration methods using an Elementar vario EL device. The intragranular porosity and the specific surface area of the samples were determined by nitrogen adsorption using a Micrometrics ASAP 2000 device. The intragranular porosity was determined at a relative pressure of $p / p_{0}=0.99$, which corresponds to a pore-diameter equivalent of $<200 \mathrm{~nm}$. These analyses were performed to relate physicochemical properties of the sediments to the sorption behavior of Raz and Rru. The characteristics of the sediments are displayed in Table 1.

\subsection{Setup of batch experiments}

Batch experiments were conducted to obtain sorption isotherms of Raz and Rru. The sediments were dried prior to the experiments to allow for a defined solid-to-liquid ratio in the batch reactors. We used $50 \mathrm{~mL}$ glass bottles filled with $30 \mathrm{~mL}$ water and $35 \mathrm{~g}$ sediment. Since Raz would quickly react to Rru in the samples under natural conditions, the samples were sterilized by $\gamma$-radiation using a specific energy dose of $10 \mathrm{kGy}$ in order to inhibit the reaction from Raz to Rru. The dose of $10 \mathrm{kGy}$ has been reported to be a good compromise between altering effects of the physical sediment properties and the sterilization efficiency (Östlund et al., 1989; Herbert et al., 2005). For each sediment, we prepared samples with Raz concentrations of $0,1,10,50$, 100 and $500 \mu \mathrm{g} \mathrm{L}^{-1}$ and Rru concentrations of $0,1,10,30$, 70 , and $100 \mu \mathrm{g} \mathrm{L}^{-1}$, both at $\mathrm{pH} 7$ and $\mathrm{pH} 9$ (adjusted with $20 \mathrm{mM}$ TAPS and $20 \mathrm{mM}$ MOPS buffer, respectively). All samples were prepared in triplicates. After preparation, all samples were shaken for approximately $40 \mathrm{~h}$ at a constant temperature of $20^{\circ} \mathrm{C}$. Subsequently, parts of the supernatant of each sample were filtered $(0.45 \mu \mathrm{m}$ glass fiber filter) and the tracer concentrations were measured by a spectrofluorometer (HORIBA Fluoro-Max-4). The concentration $s_{i}$ of the sorbed tracer per mass of solids was calculated by the difference between the initial tracer concentration and the tracer concentration in the aqueous phase after exposure to the sediments over $40 \mathrm{~h}$, normalized by the ratio of solid mass to liquid volume in the samples. The dependence of the sorbed to the dissolved tracer mass was fitted by the standard linear and Freundlich sorption models (Grathwohl, 1998).

\subsection{Setup of column experiments}

All experiments were conducted at a constant temperature of $20^{\circ} \mathrm{C}$ to avoid corrections of tracer signals due to temperature fluctuations. All devices and solutions used in the tests were stored in the same room until they reached equilibrium with the room temperature. Except for joints and connections, all tubings were made of stainless steel to avoid sorption of the tracers onto the wall of the tubes.

We used $30 \mathrm{~cm}$ long glass columns with an inner radius of $2.5 \mathrm{~cm}$ (total volume of $590 \mathrm{~cm}^{3}$ ). The columns were
Table 1. Characteristics of the sediments used in the experiments.

\begin{tabular}{lll}
\hline & Steinlach & Goldersbach \\
\hline Grain sizes & $0.08-4 \mathrm{~mm}$ & $0.08-4 \mathrm{~mm}$ \\
Grain density & $2.60 \mathrm{~kg} \mathrm{~L}^{-1}$ & $2.58 \mathrm{~kg} \mathrm{~L}^{-1}$ \\
Organic carbon content $^{*}$ & $0.73 \%$ & $0.13 \%$ \\
$\mathrm{CaCO}_{3}$ content* & $66.0 \%$ & $8.9 \%$ \\
Specific surface area & $9.92 \mathrm{~m}^{2} \mathrm{~g}^{-1}$ & $18.45 \mathrm{~m}^{2} \mathrm{~g}^{-1}$ \\
Intragranular porosity & 0.04 & 0.06 \\
\hline
\end{tabular}

* Contents are stated as percent by weight.

manually filled with sediment under water to avoid gas entrapment in the pore space. The sediment itself was untreated, i.e. not sterilized and collected analogously to the sediment for the batch experiments described above. We estimated the effective porosity by fitting the measured fluorescein BTC of each column experiment to the one-dimensional analytical solution of the advection-dispersion equation. In this framework, the porosity is estimated as the specific discharge ("Darcy velocity"), divided by the effective velocity of the tracer. The results indicate that the mean porosity of all columns was 0.45 with only small deviations between the different experiments $(\sigma=0.07, n=4)$. Both connection threads of the column were filled with glass wool to prevent particles from entering the fluorometers. A $\sim 1 \mathrm{~mm}$ thick, highly porous glass disc and a layer of $\sim 2 \mathrm{~cm}$ of pure quartz sand was placed at each end of the column to enforce parallel flow and advective tracer transport through the column (Fig. 1). The filled columns were shielded from light using aluminum foil to avoid photodegradation of the fluorescent tracers. Prior to every experiment, the columns were flushed with a solution of modified tap water at the respective $\mathrm{pH}$ (see below), until the $\mathrm{pH}$ at the outlet of the column was identical to the $\mathrm{pH}$ of the injected solution.

Fluorescein and Raz were mixed in a ratio of about $1: 10$ in approximately $10 \mathrm{~L}$ of water and poured into a glass container. This container was placed above the column so that a constant small overpressure was generated within the tubing system, which prevented air from invading the system. A peristaltic pump was placed at the outlet of the column to maintain a constant volumetric flow rate of $24 \mathrm{~mL} \mathrm{~min}^{-1}$, which was monitored throughout all experiments. Once the concentrations of all three tracers reached a maximum and remained constant at the outlet of the column, the injection solution was switched to water (at the same $\mathrm{pH}$ as the respective tracer solution).

The tracer solution in the reservoir container was prepared with tap water and adjusted to the respective $\mathrm{pH}$ using MOPS sodium salt (for $\mathrm{pH}$ 7) and TAPS sodium salt (for $\mathrm{pH}$ 9) buffers. Both buffers are frequently used in biochemical applications (Cartwright et al., 2000; Ettwig et al., 2010), and we did not expect any effects on the sorption or transformations of Raz and Rru by these buffers, except for the pure $\mathrm{pH}$ 


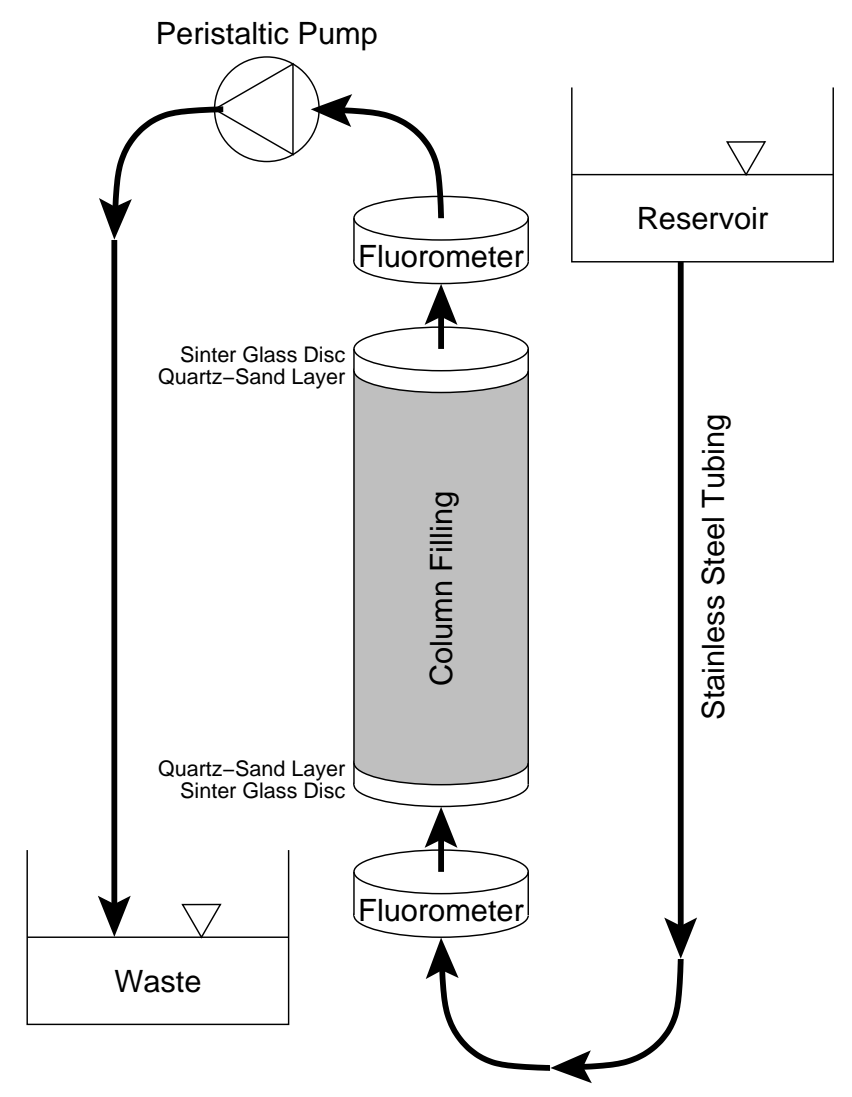

Figure 1. Schematic of the column experiments used to investigate the sorption of Raz and Rru.

effects. Nonetheless, their concentrations were kept as low as $20 \mathrm{mM}$ in all solutions to minimize possible effects by the resulting increase of the ionic strength. Just like the columns, the reservoir container was wrapped in aluminum foil to prevent photodegradation of the tracers.

We placed portable field fluorometers of the type GGUNFL30 at the inlet and outlet of the column to measure the concentrations of Raz, Rru, and fluorescein (Lemke et al., 2013b) so that they acted as flow-through cells with a volume of $16 \mathrm{~mL}$ each. Both fluorometers were calibrated prior to each column experiment to achieve the highest possible accuracy of the instruments. The instrumental sampling interval was $\leq 40 \mathrm{~s}$, which is significantly higher than in other laboratory studies using Raz and Rru (10-28 min, Stanaway et al., 2012; 15-130 min, Haggerty et al., 2008). The high temporal resolution of the concentration measurements allowed a detailed analysis of the rising and falling limb of the BTCs, which contain key information regarding sorption processes. Each experiment lasted about 4-5 h (depending on the time until steady-state conditions had been reached).

\subsection{Mathematical model for reactive transport}

The sorption parameters of Raz and Rru were determined by fitting a travel-time-based analytical model to the measured BTCs of the tracers in the column experiments. The model considers the formation of Rru as a daughter compound of Raz, decay of Raz and Rru to undetected compounds and mass retention of Raz and Rru due to equilibrium and kinetic sorption processes (Liao et al., 2013).

For the ease of description, we assume in the next paragraphs that the aqueous-phase physical transport of fluorescein, Raz, and Rru within the column may be described by the widely used advection-dispersion equation, amended by reaction and sorption terms for Raz and Rru:

$$
\begin{aligned}
& \frac{\partial c_{0}}{\partial t}+v \frac{\partial c_{0}}{\partial x}-D \frac{\partial^{2} c_{0}}{\partial x^{2}}=0 \\
& \left(K_{1}^{\mathrm{eq}}+1\right) \frac{\partial c_{1}}{\partial t}+K_{1}^{\mathrm{kin}} \frac{\partial c_{1, \mathrm{kin}}}{\partial t}+v \frac{\partial c_{1}}{\partial x}-D \frac{\partial^{2} c_{1}}{\partial x^{2}} \\
& =\left(-\lambda_{1}-\lambda_{12}\right) c_{1} \\
& \left(K_{2}^{\mathrm{eq}}+1\right) \frac{\partial c_{2}}{\partial t}+K_{2}^{\mathrm{kin}} \frac{\partial c_{2, \mathrm{kin}}}{\partial t}+v \frac{\partial c_{2}}{\partial x}-D \frac{\partial^{2} c_{2}}{\partial x^{2}} \\
& =+\lambda_{12} c_{1}-\lambda_{2} c_{2} \\
& \frac{\partial c_{i, \text { kin }}}{\partial t}=k_{i}\left(c_{i}-c_{i, \text { kin }}\right), \quad i=1,2,
\end{aligned}
$$

subject to the following boundary and initial conditions:

$$
\begin{aligned}
& c_{0}(x=0, t)=c_{\text {in }, 0}(t) \\
& c_{1}(x=0, t)=c_{\text {in }, 1}(t) \\
& c_{2}(x=0, t)=0 \\
& c_{i}(x, t=0)=0 \quad i=0,1,2 \\
& c_{i, \operatorname{kin}}(x, t=0)=0 \quad i=1,2,
\end{aligned}
$$

in which $t[\mathrm{~T}]$ is time, and $c_{i}\left[\mathrm{ML}^{-3}\right]$ is the molar aqueousphase concentration of compound $i$, with $i=0$ denoting fluorescein, $i=1 \mathrm{Raz}$, and $i=2$ Rru. $v\left[\mathrm{~L} \mathrm{~T}^{-1}\right]$ and $D\left[\mathrm{~L}^{2} \mathrm{~T}^{-1}\right]$ are the effective velocity and the dispersion coefficient, respectively. $c_{\mathrm{in}, 0}\left[\mathrm{ML}^{-3}\right]$ and $c_{\mathrm{in}, 1}\left[\mathrm{ML}^{-3}\right]$ are the concentrations of fluorescein and Raz in the inflow, respectively, whereas the inflow concentration of Rru is considered zero. $K_{i}^{\mathrm{eq}}=K_{\mathrm{d}, i}^{\mathrm{eq}} \rho_{\mathrm{b}} / \theta[-]$ and $K_{i}^{\mathrm{kin}}=K_{\mathrm{d}, i}^{\mathrm{kin}} \rho_{\mathrm{b}} / \theta[-]$ are the dimensionless distribution coefficient for the equilibrium sorption and kinetic sorption sites, respectively, whereas $K_{\mathrm{d}, i}^{\mathrm{eq}}$ and $K_{\mathrm{d}, i}^{\text {kin }}\left[\mathrm{M}^{-1} \mathrm{~L}^{3}\right]$ are the corresponding dimensional distributions coefficients. $\rho_{\mathrm{b}}\left[\mathrm{ML}^{-3}\right]$ is the bulk density of the dry soil, $\theta[-]$ denotes effective porosity, $k_{i}\left[\mathrm{~T}^{-1}\right]$ is the masstransfer rate coefficient of kinetic sorption, $\lambda_{1}\left[\mathrm{~T}^{-1}\right]$ is the rate coefficient of Raz transformation to undetected products, $\lambda_{12}\left[\mathrm{~T}^{-1}\right]$ is the transformation rate coefficient of Raz to Rru and $\lambda_{2}\left[\mathrm{~T}^{-1}\right]$ is the rate coefficient of Rru transformation to undetected products. $c_{i, \text { kin }}\left[\mathrm{ML}^{-3}\right]$ is the concentration of the sorbed tracer compound $i$ expressed as the corresponding equilibrium aqueous concentration 
$c_{i, \mathrm{kin}}=\frac{s_{i, \mathrm{kin}}}{K_{\mathrm{d}, i}^{\mathrm{kin}}}$,

in which $s_{i, \text { kin }}\left[\mathrm{M} \mathrm{M}^{-1}\right]$ is the mass-related concentration of the kinetically sorbed compound $i$.

We chose to express the effect of the equilibrium sorption by $K_{i}^{\mathrm{eq}}$ rather than a retardation factor $R_{i}$ or the dimensional distribution coefficient $K_{\mathrm{d}, i}^{\mathrm{eq}}$ to make a direct comparison to $K_{i}^{\mathrm{kin}}$ possible. If desired, the values of $K_{i}^{\mathrm{eq}}$ can easily be transferred to likewise dimensionless retardation factors by $R_{i}=K_{i}^{\mathrm{eq}}+1$.

It is known that the advection-dispersion equation shows deficiencies in exactly reproducing conservative transport, even in homogeneous porous media, whereas transport equations that show similarities to the advection-dispersion equation with a kinetic sorption term lead to better agreements with observed BTCs (Cortis et al., 2004). In this context, fitting the measured BTCs to Eqs. (1)-(2) may lead to biased results, because the fraction of tailing in the BTCs of Raz and Rru that belongs to non-Fickian conservative transport is misinterpreted as kinetic sorption. We therefore use a formulation of linear reactive transport that relies on the probability density function $g_{0}(\tau)\left[\mathrm{T}^{-1}\right]$ of travel times $\tau$ [T] rather than the advection-dispersion equation. Transport of all compounds can be expressed by convolution of the concentration in the inflow with a transfer function, because the governing transport processes are linear with respect to tracer concentrations. The transfer functions of Raz and Rru can be derived from the probability density function $g_{0}(\tau)$ of travel times and the sorption/transformation parameters listed above. For the derivation of the equations in a traveltime framework, we closely follow the concept and the notation presented by Liao et al. (2013).

Because under the given $\mathrm{pH}$ conditions fluorescein is considered to behave conservatively (Smith and Pretorius, 2002; Kasnavia et al., 1999), the BTC $c_{\text {out,Flu }}\left[\mathrm{ML}^{-3}\right]$ of fluorescein in the outflow is related to the travel-time distribution through the column $g_{0}(\tau)\left[\mathrm{T}^{-1}\right]$ and the fluorescein concentration $c_{\text {in,Flu }}\left[\mathrm{ML}^{-3}\right]$ by the following convolution integral:

$c_{\text {out }, \text { Flu }}(t)=\int_{0}^{\infty} g_{0}(\tau) c_{\text {in,Flu }}(t-\tau) \mathrm{d} \tau$,

in which $t[\mathrm{~T}]$ is the time since the start of the injection and $\tau[\mathrm{T}]$ is the residence time in the column. $g_{0}(\tau)$ is also the transfer function of fluorescein and is estimated in the present study by non-parametric deconvolution (Cirpka et al., 2007).

The transport of Raz and Rru through the column is modified by equilibrium and kinetic sorption as well as decay processes. Rru was not injected into the column but may occur in the injected solution due to the impurity of the supplied Raz stock. To calculate the concentrations of Raz and Rru at the outlet of the column, we can convolute the respective input signal of $\operatorname{Raz} c_{\mathrm{in}, 1}\left[\mathrm{ML}^{-3}\right]$ with the transfer functions $g_{1}(\tau)\left[\mathrm{T}^{-1}\right]$, expressing the response of Raz in the outlet due to a unit pulse of Raz in the inlet, $g_{12}(\tau)\left[\mathrm{T}^{-1}\right]$, expressing the response of Rru in the outlet due to a unit pulse of Raz in the inlet, and $g_{2}(\tau)\left[\mathrm{T}^{-1}\right]$, expressing the response of Rru in the outlet due to a unit pulse of Rru in the inlet, by

$$
\begin{aligned}
c_{\text {out }, 1}(t) & =\int_{0}^{\infty} g_{1}(\tau) c_{\text {in }, 1}(t-\tau) \mathrm{d} \tau \\
c_{\text {out }, 2}(t) & =\int_{0}^{\infty}\left(g_{12}(\tau) c_{\text {in }, 1}(t-\tau)+g_{2}(\tau) c_{\text {in }, 2}(t-\tau)\right) \mathrm{d} \tau .
\end{aligned}
$$

As discussed in the following, we solve transport in the travel-time domain rather than the spatial domain. This has the advantage that the validity of the estimated sorption and transformation parameters does not require that conservative transport exactly meet the advection-dispersion equation. The outlet simply samples a conservative travel-time distribution, expressed by $g_{0}(\tau)$, which implies that the transfer functions $g_{1}(\tau), g_{12}(\tau)$, and $g_{2}(\tau)$ of Raz and Rru at the outlet are weighted averages (or Fredholm integrals) of the transfer functions for all travel times $\tau_{*}$ sampled by the outlet

$$
\begin{aligned}
g_{1}(\tau)= & \int_{0}^{\infty} g_{0}\left(\tau_{*}\right) c_{1}\left(\tau, \tau_{*}\right) \mathrm{d} \tau_{*} \\
g_{12}(\tau) & =\int_{0}^{\infty} g_{0}\left(\tau_{*}\right) c_{12}\left(\tau, \tau_{*}\right) \mathrm{d} \tau_{*} \\
g_{2}(\tau)= & \int_{0}^{\infty} g_{0}\left(\tau_{*}\right) c_{22}\left(\tau, \tau_{*}\right) \mathrm{d} \tau_{*},
\end{aligned}
$$

in which $c_{i}\left(\tau, \tau_{*}\right)\left[\mathrm{T}^{-1}\right]$ is the concentration response of $\mathrm{Raz}$ and Rru to a pulse of Raz at travel time $\tau_{*}$ and time $\tau$ since the pulse release. The governing equations for $c_{i}\left(\tau, \tau_{*}\right)$ read as

$$
\begin{aligned}
& \left(K_{1}^{\mathrm{eq}}+1\right) \frac{\partial c_{1}}{\partial \tau}+K_{1}^{\mathrm{kin}} \frac{\partial c_{1, \mathrm{kin}}}{\partial \tau}+\frac{\partial c_{1}}{\partial \tau_{*}}=-\left(\lambda_{1}+\lambda_{12}\right) c_{1} \\
& \left(K_{2}^{\mathrm{eq}}+1\right) \frac{\partial c_{2}}{\partial \tau}+K_{2}^{\mathrm{kin}} \frac{\partial c_{2, \mathrm{kin}}}{\partial \tau}+\frac{\partial c_{2}}{\partial \tau_{*}}=\lambda_{12} c_{1}-\lambda_{2} c_{2}
\end{aligned}
$$

and

$\frac{\partial c_{i, \mathrm{kin}}}{\partial \tau}=k_{i}\left(c_{i}-c_{i, \mathrm{kin}}\right)$

subject to

$$
\begin{aligned}
& c_{1}\left(\tau, \tau_{*}=0\right)=b_{1} \delta(\tau) \\
& c_{2}\left(\tau, \tau_{*}=0\right)=b_{2} \delta(\tau) \\
& c_{i}\left(\tau=0, \tau_{*}>0\right)=0 \forall i,
\end{aligned}
$$


in which $\delta(\cdot)$ is the Dirac delta function with inverse units of the argument. The dimensionless coefficients $b_{1}$ and $b_{2}$ can either be zero or one and represent how much mass of the parent and daughter compounds are introduced into the columns at time $\tau=0 . c_{1}\left(\tau, \tau_{*}\right)\left[\mathrm{T}^{-1}\right]$ is the concentration of Raz caused by injection of Raz into the system for $b_{1}=1$ in Eq. (9), $c_{12}\left(\tau, \tau_{*}\right)\left[\mathrm{T}^{-1}\right]$ is the concentration of Rru caused by transformation from Raz at conservative travel time $\tau_{*}$ time $\tau$ stemming from the input of $c_{\text {in, } 1}$, that is for $b_{1}=1$ and $b_{2}=0$ in Eq. 9 , and $c_{22}\left(\tau, \tau_{*}\right)\left[\mathrm{T}^{-1}\right]$ is the concentration of Rru caused by injection of the same compound into the column, stemming from the input of $c_{i n, 2}$ (that is, for $b_{1}=0$ and $b_{2}=1$ ) in Eq. (9). Note that in Eqs. (6)-(9) $c_{i}$ and $c_{i \text {,kin }}$ have units of inverse times, because they are not actual concentrations, different from Eqs. (1)-(3), in which convolution with input signals is not performed.

Equations (7)-(9) can conveniently be solved in the Laplace domain:

$\tilde{c}_{1}\left(s, \tau_{*}\right)=\exp \left(-\beta_{1} \tau_{*}\right)$

$\tilde{c}_{2}\left(s, \tau_{*}\right)=d_{2} \exp \left(-\beta_{2} \tau_{*}\right)+d_{12} \exp \left(-\beta_{1} \tau_{*}\right)$,

in which the $\tilde{c}$ denotes the Laplace transform with respect to $\tau, s$ is the Laplace variable, and the coefficients are defined as (Liao et al., 2013)

$$
\begin{aligned}
& \beta_{1}=\left(1+K_{1}^{\mathrm{eq}}\right) s+\lambda_{1}+\lambda_{12}+K_{1}^{\mathrm{kin}} s \frac{k_{1}}{s+k_{1}} \\
& \beta_{2}=\left(1+K_{2}^{\mathrm{eq}}\right) s+\lambda_{2}+K_{2}^{\mathrm{kin}} s \frac{k_{2}}{s+k_{2}} \\
& d_{2}=\frac{\lambda_{12}}{\beta_{1}-\beta_{2}} \\
& d_{12}=-\frac{\lambda_{12}}{\beta_{1}-\beta_{2}} .
\end{aligned}
$$

Equation (11) is back-transformed into the time domain by the numerical method of De Hoog et al. (1982).

\subsection{Parameter inference and uncertainty estimation}

A Bayesian approach was adopted in this study to quantify model parameters and their uncertainty. The methods are identical to our previous work (Lemke et al., 2013a; Wöhling et al., 2012) and therefore we subsequently include only a brief summary of the approach.

Let us consider the transport model $f$ that simulates the Raz and Rru concentrations summarized as system response $\boldsymbol{Y}=\left\{y_{1}, \ldots, y_{n}\right\}$ with length $n$ using a vector of $m=9$ model parameters, $\boldsymbol{u}=\left\{u_{1}, \ldots, u_{m}\right\}: \boldsymbol{Y}=f(\boldsymbol{u})$. Further, we consider that $\tilde{\boldsymbol{Y}}$ denotes a vector with the observed concentration data. We then combine the data likelihood, $p(\boldsymbol{u} \mid \tilde{\mathbf{Y}})$ with a prior distribution $p(\boldsymbol{u})$ by the Bayes' theorem to infer the posterior probability density function $(p d f)$ of the model parameter vector $\boldsymbol{u}$ :

$$
p(\boldsymbol{u} \mid \tilde{\mathbf{Y}}) \propto p(\tilde{\mathbf{Y}} \mid \boldsymbol{u}) p(\boldsymbol{u})
$$

We assume measurement errors, $\sigma_{\mathrm{Raz}}=2.23 \mu \mathrm{mol} \mathrm{m}{ }^{-3}$ and $\sigma_{\text {Rru }}=0.12 \mu \mathrm{mol} \mathrm{m}^{-3}$ for Raz and Rru concentrations, respectively, which accounts for the higher uncertainty in the measurements of Raz due to its lower quantum yield. We further assume the error residuals to be uncorrelated and normally distributed with constant variance, and replace the data likelihood function $p(\boldsymbol{u} \mid \tilde{\mathbf{Y}})$ by an aggregated likelihood function, $\ell(\boldsymbol{u} \mid \tilde{\mathbf{Y}})$, for the joint fitting of the Raz and Rru breakthrough curves:

$$
\begin{aligned}
\ell(\boldsymbol{u} \mid \tilde{\mathbf{Y}})= & -\frac{n_{\mathrm{Raz}}}{2} \ln (2 \pi)-\frac{n_{\mathrm{Raz}}}{2} \ln \left(\sigma_{\mathrm{Raz}}^{2}\right) \\
& -\frac{1}{2} \sum_{j=1}^{n_{\mathrm{Raz}}} \frac{\left(y_{\mathrm{Raz}, j}(\boldsymbol{u})-\tilde{y}_{\mathrm{Raz}, j}\right)^{2}}{\sigma_{\mathrm{Raz}}^{2}} \\
& -\frac{n_{\mathrm{Rru}}}{2} \ln (2 \pi)-\frac{n_{\mathrm{Rru}}}{2} \ln \left(\sigma_{\mathrm{Rru}}^{2}\right) \\
& -\frac{1}{2} \sum_{j=1}^{n_{\mathrm{Rru}}} \frac{\left(y_{\mathrm{Rru}, j}(\boldsymbol{u})-\tilde{y}_{\mathrm{Rru}, j}\right)^{2}}{\sigma_{\mathrm{Rru}}^{2}},
\end{aligned}
$$

where $y_{\operatorname{Raz}, j}(\boldsymbol{u})$ and $y_{\mathrm{Rru}, j}(\boldsymbol{u})$ are the model-predicted values for Raz and Rru, respectively, and $\tilde{y}_{\mathrm{Raz}, j}\left(j=1, \ldots, n_{\mathrm{Raz}}\right)$ and $\tilde{y}_{\mathrm{Rru}, j}\left(j=1, \ldots, n_{\mathrm{Rru}}\right)$ are the corresponding observations. The parameter vector utilized in Eq. (12) is $\boldsymbol{u}=\left\{K_{i}^{\mathrm{eq}}, K_{i}^{\mathrm{kin}}, k_{i}, \lambda_{1}, \lambda_{12}, \lambda_{2}\right\}$.

The prior distribution, $p(\boldsymbol{u})$ was assumed to be uniform with the following parameter ranges: $R_{1}, R_{2}=\left[\begin{array}{l}1 \\ \ldots\end{array}\right]$; $K_{1}^{\mathrm{kin}}, K_{2}^{\mathrm{kin}}=[0 \ldots 3] ; k_{1}, k_{2}=\left[1 \times 10^{-6} \ldots 1 \times 10^{-2}\right]$; and $\lambda_{1}, \lambda_{12}, \lambda_{2}=\left[1 \times 10^{-7} \ldots 1 \times 10^{-2}\right]$. The choice of these ranges was guided by the preliminary data analysis and previously published values (Haggerty et al., 2008; Liao et al., 2013).

To generate samples from the posterior distribution, we use the differential evolution adaptive metropolis $\left(\right.$ DREAM $_{\mathrm{ZS}}$ ) adaptive Markov chain-Monte Carlo (MCMC) scheme. The convergence of the DREAM ${ }_{Z S}$ runs was monitored by the $\hat{R}$ statistic of Gelman and Rubin (1992). In our calculations, we used $n=10$ Markov chains and selected the last 10000 accepted samples (after convergence was observed in all chains) for the calculation of the posterior parameter $p d f$ s. All other algorithmic parameters are set to their recommended values. For more details on the parameter inference scheme, please refer to ter Braak and Vrugt (2008), Wöhling and Vrugt (2011), Schoups and Vrugt (2010), and Wöhling et al. (2012).

\section{Results and discussion}

\subsection{Batch experiments}

The batch experiments were conducted in order to obtain equilibrium sorption isotherms for Raz and Rru. Figure 2 shows the mass-related concentrations of the sorbed tracers as function of the aqueous-phase concentration after equilibration. The complete data set of all replicates is available in 

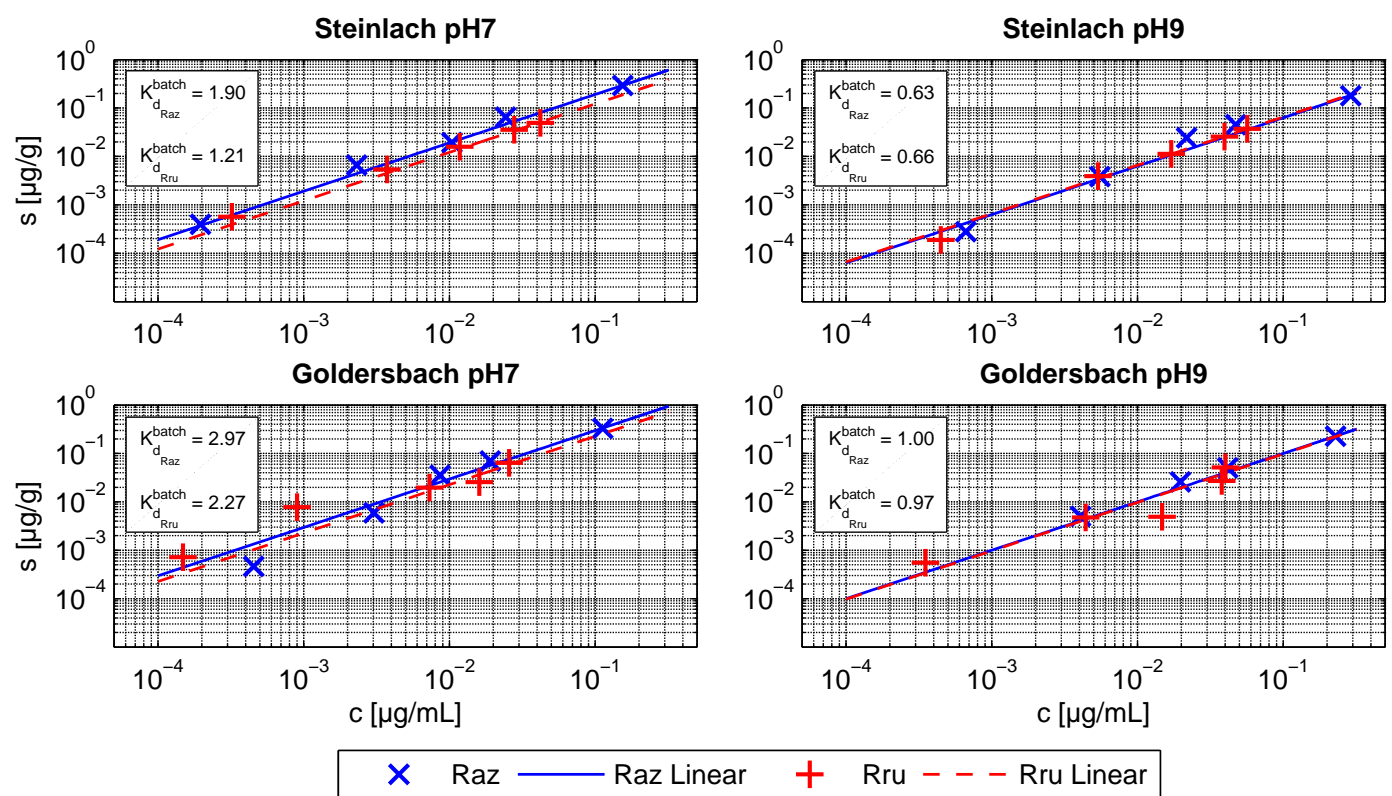

Figure 2. Linear sorption isotherms of Raz and Rru for the different $\mathrm{pH}$ values and sediment types. Outliers of replicate measurements are not shown for clarity. The complete data sets are available in the Supplement. Values of $K_{\mathrm{d}_{i}}^{\text {batch }}$ in $\mathrm{L} \mathrm{kg}^{-1}$.

the Supplement. The dashed lines represent linear sorption isotherms, which are in good agreement with the observed data (mean RMSE value of $5.68 \times 10^{-3} \mu \mathrm{g} \mathrm{mL}^{-1}$ ). Applying a Freundlich isotherm did not result in significantly better overall fits (mean RMSE $=3.03 \times 10^{-3} \mu \mathrm{g} \mathrm{mL}^{-1}$, curves not shown), so that we assume that linear sorption (as described by the single parameter $K_{\mathrm{d}_{i}}^{\text {batch }}\left[\mathrm{L}^{3} \mathrm{M}^{-1}\right]$ ) is adequate for the interpretation of field tracer tests for concentrations of up to $500 \mu \mathrm{g} \mathrm{L}^{-1}$ of Raz and $100 \mu \mathrm{g} \mathrm{L}^{-1} \mathrm{Rru}$. We did not measure the concentrations of Raz and Rru over time in the batch experiments, because any information about kinetic sorption processes would be incomparable to the column tests due to the different solid-to-water ratios and the continuous shaking of the batch samples. Thus, the values of $K_{\mathrm{d}_{i}}^{\text {batch }}$ represent the distribution of the tracers between the liquid phase and the sum of all sorption sites.

The $K_{\mathrm{d}_{i}}^{\text {batch }}$ values for Raz and Rru are almost identical at pH 9 (Steinlach: 0.63 and $0.66 \mathrm{~L} \mathrm{~kg}^{-1}$, respectively, and Goldersbach: 1.00 and $0.97 \mathrm{~L} \mathrm{~kg}^{-1}$, respectively) for the individual sediments. At $\mathrm{pH} 7$, the $K_{\mathrm{d}_{i}}^{\text {batch }}$ values are generally higher compared to those at $\mathrm{pH} \mathrm{9,} \mathrm{and} \mathrm{sorption} \mathrm{of} \mathrm{Raz} \mathrm{ap-}$ pears to be stronger than the sorption of Rru. This is consistent with the $p K_{\mathrm{a}}$ values of Raz (6.7) and Rru (5.7): at $\mathrm{pH} 7$, about one-third of the Raz molecules are in the acidic form, whereas more than $95 \%$ of the Rru molecules remain as anions.

The values obtained for $K_{\mathrm{d}_{i}}^{\text {batch }}$ would result in retardation factors of up to 20 within sediments, which is much higher compared to retardation factors of Raz and Rru reported in other studies (Lemke et al., 2013b; Liao et al.,
2013). A possible reason for this discrepancy may be that batch experiments tend to overestimate sorption (Grolimund et al., 1995). It is known that the conditions in batch reactors usually differ from those in the field - i.e., due to higher water-to-solid ratios or to discarding the smallest and largest grain sizes. An alternative (and probably more evident) reason for our findings lies in the way the $K_{\mathrm{d}_{i}}^{\text {batch }}$ values were obtained. The calculation of the $K_{\mathrm{d}_{i}}^{\text {batch }}$ values is based on the premise that the removal of tracer substances from the aqueous phase in the samples is solely caused by sorption. However, both Raz and Rru may undergo chemical transformation processes, which also results in an effective removal of the tracers. These processes are not accounted for in the standard linear sorption model as applied in the analysis of the batch experiments, because it is impossible to distinguish between decay and sorption as reasons for tracer removal from the aqueous phase. Therefore, the resulting $K_{\mathrm{d}_{i}}^{\text {batch }}$ values describe the maximum partition of the tracers between the solids and the liquid (i.e., these values are only valid when no decay of Raz and Rru occurs during the sorption experiment) (Haggerty et al., 2008).

We found that a certain amount of Rru was formed in the batch samples that had a pH of 7 (on average $6 \%$ of the initial molar Raz concentration), although the combination of the exposure of high temperatures $\left(60^{\circ} \mathrm{C}\right.$ while drying the sediments for several days) and $\gamma$-radiation should have destroyed all living cells and also possible remains of enzymatic structures. We thus cannot guarantee that the sediments were entirely sterile, so that possibly a small number of living bacteria had enough time to transform a detectable amount 
of Raz under the advantageous neutral $\mathrm{pH}$ conditions in the batch reactors. However, the fact that decay processes occurred in the near-sterile sediments shows that they may not be triggered only by microbial activity.

Unfortunately, we found that the long exposure to the sediments altered the $\mathrm{pH}$ in the samples due to the buffer capacity of the carbonate balance. The $\mathrm{pH}$ of the $\mathrm{pH} 7$ samples increased to 7.4 and the $\mathrm{pH}$ of the $\mathrm{pH} 9$ samples decreased to 8.2 after the exposure to the sediments. However, the difference in $\mathrm{pH}$ between the samples is still big enough, so that the conclusions drawn from the batch experiments with respect to the $\mathrm{pH}$ remain unaffected.

\subsection{Column experiments}

Figure 3 shows the transfer functions of fluorescein, Raz, and Rru derived from Eqs. (4) to (6) for the column with the Goldersbach sediment at $\mathrm{pH}$ 7. The corresponding parameters were obtained by fitting the observed BTCs. Similar transfer functions were obtained for the other column experiments. In all cases, the transfer function of the conservative tracer fluorescein represents the travel-time distribution of water, because fluorescein is known to behave like an ideal tracer at the given $\mathrm{pH}$. The mean residence time of water in the column (mean of the distribution) ranges from 9 to $12 \mathrm{~min}$. The transfer functions of Raz and Rru are delayed against fluorescein due to sorption, which also produces a pronounced tailing in the transfer functions of the reactive tracers. While the travel-time distribution of fluorescein resembles a lognormal distribution, a closer analysis reveals some non-Fickian tailing, which could not be detected by fitting an advective-dispersive transport model to the data but is revealed by non-parametric deconvolution of the fluorescein in- and output signals (Cirpka et al., 2007).

Figure 4 shows the observed BTCs and the model fits obtained by the most likely parameters for all tracers in the four column experiments. The measured data are plotted as markers, but the temporal resolution was so high that they appear as continuous lines. Note that only fluorescein and Raz were injected, whereas the measured Rru originates solely from the Raz-to-Rru reaction. The modeled curves fit the measured data very well in all cases (RMSE between $3.8 \times 10^{-4}$ and $6.7 \times 10^{-3} \mu \mathrm{mol} \mathrm{L}^{-1}$ ). One exception is the fit for Raz for the Steinlach sediment at pH 7 (RMSE $1.0 \times 10^{-2} \mu \mathrm{mol} \mathrm{L}^{-1}$ ) where we observed small discrepancies between modeled and measured data at medium to high concentrations in parts of the rising and falling limbs. We attribute these differences to technical problems of the fluorometer, and thus neglected the respective parts in the fitting of the BTCs.

All plots of Fig. 4 contain subplots showing the same BTCs in a semilogarithmic scale to highlight the tails of the BTCs. Apparently, there is a very good agreement between measured and simulated data for fluorescein in all four column experiments, even in semilogarithmic scale. This is

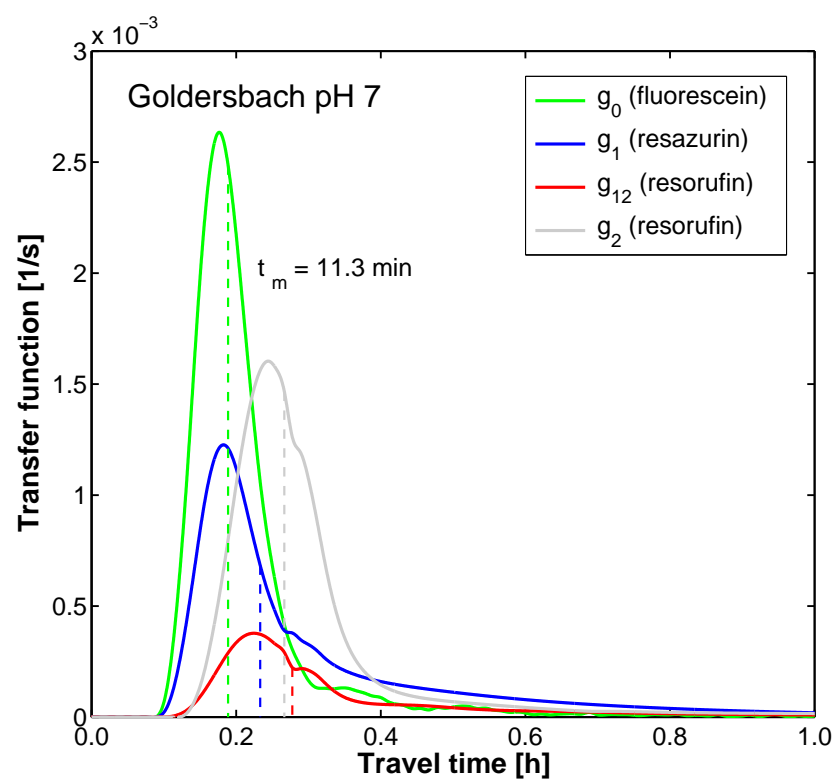

Figure 3. Transfer functions of fluorescein (Fluo), resazurin (Raz), and resorufin (Rru) for the Goldersbach sediment at $\mathrm{pH}$ 7. Similar transfer functions were obtained for the other column experiments. The mean of the transfer functions are indicated by dashed lines with that of Fluo (labeled with $t_{\mathrm{m}}$ ), representing the mean travel time of tracers through the column. The transfer function $g_{12}$ represents Rru from the transformation of Raz within the column, whereas $g_{2}$ is related to Rru already present at the column inlet, which in the case of the experiments is due to minor impurities of the Raz.

of particular importance as any model error in the conservative transport through the columns will also influence the reactive transport and most likely manifest itself as bias of the reactive transport parameters (which is often difficult to identify). The good agreement of the fluorescein data in Fig. 4 indicates that the travel-time-based model approach used here is able to adequately describe the conservative transport in the column experiments and therefore may provide reactive transport parameters of high informative value.

In contrast to the conservative tracer, the semi-log scale reveals deviations between simulated and measured Raz concentrations, though these deviations, as well as the absolute concentrations in the tails, are in general very small (less than one $\mu \mathrm{mol} \mathrm{L}^{-1}$ ). The observed extended tails of the BTCs not being correctly simulated by the models most likely indicate the presence of slow kinetic effects that are not represented by the chosen modeling approach. Accounting for these long-term processes might be possible by including intra-particle diffusion in the model. However, this would result in a further increase in model complexity with additional parameters. On the other hand, applying simpler models that neglect kinetic sorption would have resulted in larger deviations between simulated and measured BTCs, particularly at the beginning of the plateau phase. 
Table 2. Maximum-likelihood parameter values describing sorption properties obtained by the column experiments. Bottom rows: comparison between values of distribution coefficients $K_{\mathrm{d}_{i}}^{\text {column }}$ and $K_{\mathrm{d}_{i}}^{\text {batch }}$ between all sorption sites and water in the column and batch experiments.

\begin{tabular}{|c|c|c|c|c|}
\hline & \multicolumn{2}{|c|}{ Steinlach } & \multicolumn{2}{|l|}{ Goldersbach } \\
\hline & pH 7 & pH 9 & pH 7 & pH 9 \\
\hline$K_{\mathrm{Raz}}^{\mathrm{eq}}[-]$ & $0.50 \pm 0.003$ & $0.10 \pm 0.002$ & $0.06 \pm 0.01$ & $0.063 \pm 0.001$ \\
\hline$K_{\mathrm{Rru}}^{\mathrm{eq}}[-]$ & $0.002 \pm 0.001$ & $0.001 \pm 0.001$ & $0.38 \pm 0.01$ & $0.12 \pm 0.01$ \\
\hline$K_{\mathrm{Raz}}^{\mathrm{kin}}[-]$ & $0.58 \pm 0.01$ & $0.27 \pm 0.003$ & $0.58 \pm 0.003$ & $0.11 \pm 0.001$ \\
\hline$K_{\mathrm{Rru}}^{\mathrm{kin}}[-]$ & $2.79 \pm 0.04$ & $0.53 \pm 0.03$ & $2.65 \pm 0.03$ & $1.98 \pm 0.19$ \\
\hline$k_{\operatorname{Raz}}\left[\mathrm{s}^{-1}\right] \times 10^{-4}$ & $8.39 \pm 0.16$ & $8.97 \pm 0.28$ & $14.11 \pm 0.13$ & $12.78 \pm 0.21$ \\
\hline$k_{\text {Rru }}\left[\mathrm{s}^{-1}\right] \times 10^{-4}$ & $0.56 \pm 0.01$ & $11.06 \pm 0.75$ & $0.98 \pm 0.01$ & $2.29 \pm 0.17$ \\
\hline$\lambda_{1}\left[\mathrm{~s}^{-1}\right] \times 10^{-5}$ & $3.50 \pm 0.61$ & $1.43 \pm 0.18$ & $0.04 \pm 0.01$ & $4.10 \pm 0.08$ \\
\hline$\lambda_{12}\left[\mathrm{~s}^{-1}\right] \times 10^{-5}$ & $62.2 \pm 0.1$ & $7.24 \pm 0.17$ & $45.53 \pm 0.04$ & $4.73 \pm 0.01$ \\
\hline$\lambda_{2}\left[\mathrm{~s}^{-1}\right] \times 10^{-5}$ & $0.20 \pm 0.09$ & $0.3 \pm 6.3$ & $0.02 \pm 0.05$ & $0.02 \pm 0.85$ \\
\hline$K_{\mathrm{d}^{2}}^{\text {column }}\left[\mathrm{L} \mathrm{kg}^{-1}\right]$ & 0.23 & 0.05 & 0.10 & 0.04 \\
\hline$K_{\mathrm{d}_{\text {Rru }}}^{\text {column }}\left[\mathrm{L} \mathrm{kg}^{-1}\right]$ & 0.58 & 0.08 & 0.47 & 0.37 \\
\hline$\left.K_{\mathrm{d}^{\text {batch }}}^{\text {a kg }} \mathrm{Lg}^{-1}\right]$ & 1.90 & 0.63 & 2.97 & 1.00 \\
\hline$K_{\mathrm{d} \text { Rru }}^{\text {batch }}\left[\mathrm{L} \mathrm{kg}^{-1}\right]$ & 1.21 & 0.66 & 2.27 & 0.97 \\
\hline
\end{tabular}
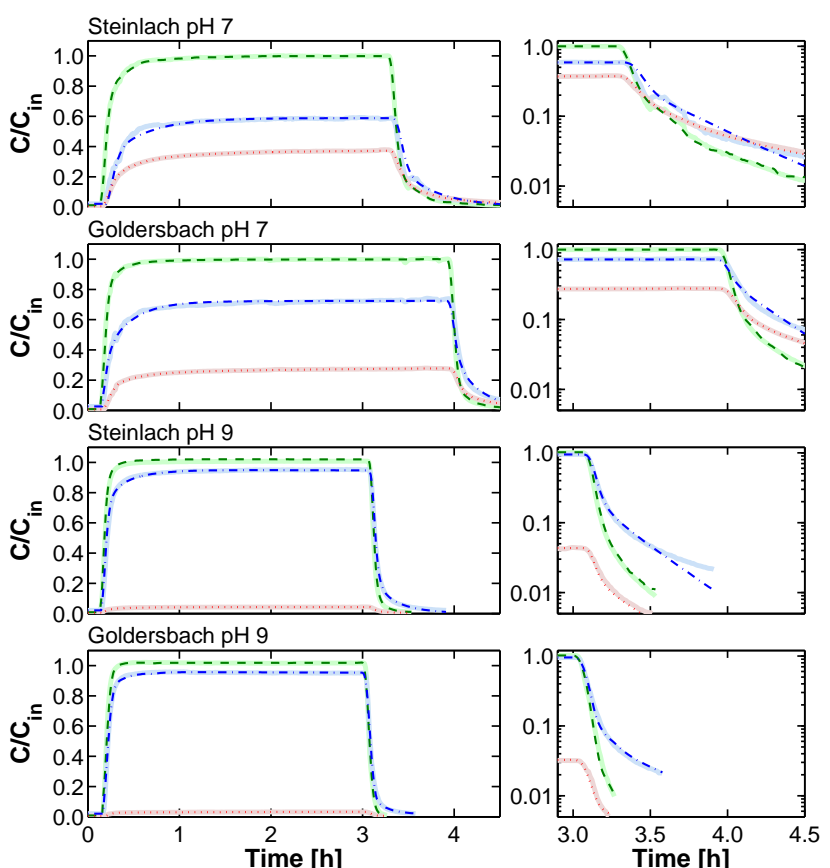

--- Fluorescein $\quad \cdots \cdot \cdots$ Resazurin $\cdots \cdots \cdot$ Resorufin $=$ Measurements

Figure 4. Measured and modeled tracer BTCs (column outlets) for Fluo, Raz and Rru at pH 7 and pH 9 and for both sediments. All tracer concentrations are normalized to the concentrations $C_{\text {in }}$ at the column inlets. Modeled BTCs of Fluo, Raz, and Rru are indicated by dashed, dash-dot, and dotted lines, respectively.
Table 2 lists the most likely values of all parameters introduced in Eqs. (6)-(8) and their associated uncertainties. The uncertainty of each parameter is quantified by the standard deviation of the posterior parameter distribution. All absolute uncertainties are generally very small. The relative errors are smaller than $10 \%$ for the parameters $R_{i}, K_{i}, k_{i}$ and $\lambda_{12}$, whereas the decay coefficients $\lambda_{1}$ and $\lambda_{2}$ have relative errors exceeding $100 \%$ in some cases. However, the estimated parameter values for these decay coefficients are extremely low $\left(0.02-4.1 \times 10^{-5} \mathrm{~s}^{-1}\right)$ and the model is not sensitive to small changes in these parameter values. An additional systematic uncertainty of the parameters in Table 2 may have resulted from the preparation of the sediments used for the experiments. Due to discarding the finest sediment fraction with potentially large reactive surface area, the sorption parameters of the reactive tracers might be underestimated in the column experiments. However, this probably does not influence the relative difference of kinetic and equilibrium sorption found in the column experiments.

As listed in Table 2, the values for $K_{i}^{\mathrm{eq}}$ at $\mathrm{pH} 9$ are very close to 0 in both sediments (which is equivalent to retardation factors close to 1 , see Sect. 2.4), suggesting that equilibrium sorption is negligible under alkaline conditions. Again, these findings agree well with the $p K_{\mathrm{a}}$ values of $\mathrm{Raz}$ and Rru, being 6.7 (Erban and Hubert, 2010) and 5.7 (Kangasniemi, 2004), respectively, implying that these compounds occur almost entirely in their anionic form at $\mathrm{pH} 9$ (see Sect. 3.1). At this $\mathrm{pH}$, the mineral surfaces of the sediments used here are most likely predominantly negatively charged, too (Hingston et al., 1972). Consequently, the electrostatic repulsion of mineral surfaces and dissolved tracers hinder sorption. At $\mathrm{pH} 7$, by contrast, equilibrium sorption may be 
important, even though not consistently among the tracers and sediments: Raz exhibits equilibrium sorption at Steinlach sediments, $R_{\text {Raz }}=1.5$, and Rru at Goldersbach sediments, $R_{\text {Rru }}=1.4$.

Kinetic sorption is more important than equilibrium sorption for both compounds in both sediments at both $\mathrm{pH}$ values, as can be seen from the distribution coefficients $K_{i}^{\text {kin }}$ between kinetic sorption sites and water listed in Table 2. Under the given $\mathrm{pH}$ conditions, the observed kinetic sorption is most likely due to diffusive transport of the reactive tracers Raz and Rru into or out of intraparticle pores of the sediment grains. Similar to equilibrium sorption, $K_{i}^{\text {kin }}$ values for alkaline conditions at $\mathrm{pH} 9$ are consistently lower than values at $\mathrm{pH} 7$, indicating that the speciation of the reactive tracers also affects the processes governing kinetic sorption. Independent of sediment type and $\mathrm{pH}$, the values of $K_{i}^{\text {kin }}$ are generally higher for Rru (0.53-2.79) than those for Raz (0.110.58). This implies that the sorption of Raz and Rru has to be parameterized independently (i.e., no common sorption parameters) in analyzing field tracer tests. The comparison of the two sediments types reveals no obvious difference in $K_{i}^{\text {kin }}$ values between the Steinlach and the Goldersbach sediments despite their small disparities in intraparticle porosity and surface area (see Table 1).

In order to compare the findings from the column experiments to the values of $K_{\mathrm{d}}^{\text {batch }}$, we computed the equivalent distribution coefficient in the column experiments $K_{\mathrm{d}}^{\text {column }}$ by

$K_{\mathrm{d}_{i}}^{\mathrm{column}}=\frac{\left(K_{i}^{\mathrm{eq}}+K_{i}^{\mathrm{kin}}\right) \theta}{\rho_{\mathrm{b}}}$,

in which $\theta[-]$ is the effective porosity in the column derived from the fluorescein data (see Sect. 2.2) and $\rho_{\mathrm{b}}\left[\mathrm{ML}^{-3}\right]$ is the bulk dry density of the sediment (see Table 1). The respective results are listed at the bottom of Table 2 .

The $K_{\mathrm{d}_{i}}^{\text {batch }}$ values significantly exceed the $K_{\mathrm{d}_{i}}^{\text {column }}$ values in all cases (Table 2), so that the sorption of the tracers appears to be generally stronger in the batch reactors than in the columns. However, the findings from the batch experiments systematically overestimate the sorption capacity of Raz and Rru (see Sect. 3.1), and it is likely that the column experiment setup better represents field conditions in stream sediments, thus providing more realistic information about sorption characteristics of the compounds.

In other studies, retardation factors between 2 and 2.5 have been found (Haggerty et al., 2009; Stanaway et al., 2012). However, these studies did not consider kinetic sorption. If we add the sorption strength of kinetic and equilibrium sites $R_{i}^{\mathrm{tot}}=K_{i}^{\mathrm{eq}}+K_{i}^{\mathrm{kin}}+1$, which is valid for transport timescales much larger than $k_{i}^{-1}$, we obtain similar numbers.

The values for the rate coefficient $k_{i}$ of kinetic sorption vary between $0.56 \times 10^{-4} \mathrm{~s}^{-1}$ and as much as $12.8 \times 10^{-4} \mathrm{~s}^{-1}$, and no evident correlations of $k_{i}$ with the sediment type or $\mathrm{pH}$ are identifiable. The inverse of $k_{i}$ is a characteristic time of sorption. In our experiments, the values for $k_{i}^{-1}$ lie between 12 and $298 \mathrm{~min}$. In comparison, the mean residence times of the tracers in the columns were about $11 \mathrm{~min}$. This implies that the quickest kinetic sorption process (Raz in Goldersbach sediments, $k_{\mathrm{Raz}}^{-1} \approx 12 \mathrm{~min}$ ) almost reaches equilibrium during the passage through the column, whereas the sorption kinetics in the other cases (Raz in Steinlach and Rru in both sediments) could not be neglected. Moreover, for Raz the typical timescales of kinetic sorption are smaller (factor 1.5 to 30) than the mean lifetime of the reaction Raz to Rru (inverse of the decay rate parameter $\lambda_{12}$ ), suggesting that kinetic sorption and desorption of Raz is relevant and should not be neglected in the experiments. This would be the case if kinetic sorption was considerably slower compared to the reaction of Raz to Rru.

The rate coefficients for decay of $\operatorname{Raz}\left(\lambda_{1}\right)$ and $\operatorname{Rru}\left(\lambda_{2}\right)$ to non-detected compounds are generally very small in all experiments (between $0.02 \times 10^{-5} \mathrm{~s}^{-1}$ and $4.1 \times 10^{-5} \mathrm{~s}^{-1}$ ) so that even in the case of the highest rate, only about $3 \%$ of the tracers had been converted to non-detected products after contact times with the sediments of about 11 min (mean travel time of the tracers through the columns). Argerich et al. (2011) reported a positive relation between organic matter content of the sediment and decay coefficients. In contrast, we could not find any clear relationship between the decay rate coefficients and the type of sediment used or the $\mathrm{pH}$ applied in our experiments.

Apparently, the reaction rate of Raz to Rru $\left(\lambda_{12}\right)$ strongly depends on $\mathrm{pH}$, which can clearly be seen by the different plateau concentrations of Rru in Fig. 4 . At $\mathrm{pH} 7, \lambda_{12}$ ranges from $45.5 \times 10^{-5} \mathrm{~s}^{-1}$ to $62.2 \times 10^{-5} \mathrm{~s}^{-1}$, which is about 1 order of magnitude higher compared to values of the same parameter at $\mathrm{pH} 9\left(4.73 \times 10^{-5} \mathrm{~s}^{-1}-7.24 \times 10^{-5} \mathrm{~s}^{-1}\right)$. Thus, at neutral conditions the reaction of Raz to Rru dominates the transformations of the Raz-Rru system, whereas at high $\mathrm{pH}$, the reaction rates of Raz to Rru are comparable to those of the decay of the tracers to non-detectable compounds for the Goldersbach sediment $\left(\lambda_{1}=4.1 \times 10^{-5} \mathrm{~s}^{-1}\right)$. While it is known that aerobic respiration depends on $\mathrm{pH}$ conditions with a decrease of respiration in the acidic $\mathrm{pH}$ range (Baker et al., 1982; Wang et al., 2006; McKinley and Vestal, 1982), the nearly uniform reduction of dissolved oxygen (between 7 and $11 \%$ relative change) measured during the column experiments did not give evidence for an influence of changes from $\mathrm{pH} 7$ to $\mathrm{pH} 9$ on the respiration rates. However, besides respiration, the dissolved oxygen at the outlet of the column may also be influenced by entrapped air within the column if the oxygen of this air component has not been completely removed by the circulating water. An alternative interpretation would be that the proportionality factor relating Raz-toRru transformation rates to respiration rates depends on $\mathrm{pH}$, which is possible because the standard biochemical pathway of $\mathrm{Raz}$ by reduction of $\mathrm{NADH} / \mathrm{H}^{+}$or $\mathrm{NADPH} / \mathrm{H}^{+}$involves an acid-base reaction. 


\section{Conclusions and recommendations}

\subsection{Implications for stream tracer tests}

We have found that Raz and Rru sorb onto sediments, which should be accounted for when interpreting tracer-test data. If not considered, the effects of sorption might misleadingly be captured by parameters that address other processes in standard modeling approaches, resulting in an erroneous characterization of hyporheic exchange and microbial activity in the hyporheic zone. The column experiments showed that under alkaline conditions ( $\mathrm{pH}$ 9) equilibrium sorption plays a minor role while kinetic sorption might still be important. In general, kinetic sorption seems to dominate sorption in local equilibrium in all our test cases on the timescale of these experiments ( $\approx 11 \mathrm{~min})$.

We have shown that, in spite of their similar molecular structure, Raz and Rru show different sorption behavior in most of our test cases, so that assuming identical sorption characteristics (as it has been done in previous studies) might be an oversimplification.

We have further shown that linear sorption of Raz and Rru is feasible for concentrations that typically occur during field tracer tests. However, we could not identify clear relations between the organic carbon content of the sediments with the sorption and decay characteristics of Raz and Rru.

We have furthermore demonstrated that the reaction rate coefficient of Raz to Rru, $\lambda_{12}$, differs between the two $\mathrm{pH}$ conditions used in this study. Respiration is likely to be different for different $\mathrm{pH}$ as well, so that the change in the reaction rate may result from a change in respiration rate, but it is beyond the scope of this study to determine the mechanisms behind the relation between $\mathrm{pH}$ and $\lambda_{12}$. Although the decay mechanisms of Raz and Rru to undetected products still ultimately remain unclear, we have found strong evidence that these reactions are not primarily driven by microbial activity.

The large relative differences of the parameters listed in Table 2 provided important insight into relevant sorption and decay processes for the sediments and timescales tested here. However, the results provided by laboratory experiments are often difficult to transfer to field sites due to the artificial geometry and conditions as well as necessary pre-treatment of the sediments. Therefore, a more detailed interpretation of the derived parameters (including relationships with sediment or physicochemical conditions) is probably not possible based on the laboratory experiments. Further work is needed to better resolve the sorption properties of Raz and Rru, which most probably has to be based on field tracer tests. This may also require refinements to the modeling approach, for example, by including intra-particle diffusion in the model instead of lumping the kinetic sorption process as two-site sorption.

For stream tracer tests we suggest (1) acknowledging that Raz and Rru have different sorption properties, (2) accounting for both kinetic and equilibrium sorption (especially when the river sediments are known to have a distinct inner porosity) and (3) checking the validity of linear sorption for studies where tracer concentrations exceed the concentrations that were used in this study. In previous studies (Lemke et al., 2013b, Liao et al., 2013) we have determined the sorption and reaction parameters together with parameters describing in-stream transport and hyporheic exchange by fitting stream tracer data to complex models. This approach leads to apparent parameters, presumably valid for the entire reach under investigation. However, we highly advise performing independent column studies like those presented here (and summarized again in the next section) in order to test the plausibility of the reactive parameters.

\subsection{Recommendations for site-specific column experiments}

The presented results show that the setup of the laboratory experiments used in this study is well suited to assess the relevant sorption and decay properties of Raz and Rru in coarse hyporheic sediments. Evidence on the linearity of sorption isotherms is provided by the batch experiments, whereas the column experiments allow for the identification of the relative importance of kinetic or equilibrium sorption as well as the relative importance of the decay of Raz to Rru as compared to the decay to unknown (non-fluorescent) compounds. The absolute parameter values derived from the laboratory experiments are most probably biased but may serve as rough orientation or order-of-magnitude estimates of the effective parameters. Detailed interpretations including interrelations with environmental variables such as $\mathrm{pH}$, organic carbon content, or sediment texture should be based on the analysis of the field tracer tests. The results of the laboratory experiments, however, are essential for selecting the adequate processes (e.g., by adding kinetic sorption) that should be included in the modeling approach for the field tracer test.

In order to gain understanding of the site-specific aspects of sorption properties of Raz and Rru, we suggest adapting the experiments to other sites where readers have performed or are planning field tracer tests of their own. The proposed column experiments are intended to be performed in conjunction (or in advance) with stream tracer tests using Raz as reactive tracer in the field. The most important issues of the experimental setup are as follows:

1. Representative sediments for the column experiments should be taken from the studied stream reach with minimal time between the collection of the sediment and the start of the experiments.

2. The sediments should be sieved in the field to grain sizes that minimize negative effects of turbidity (in our case between 0.08 and $4 \mathrm{~mm}$ ) and therefore ensure highquality fluorescence measurements and consecutively high-quality modeling. 
3. The flow velocity of water through the column should be adjusted in such a way that the mean residence time of water (and tracer) in the column matches the expected or known mean hyporheic travel time of the stream reach under investigation.

4. Besides the reactive tracer Raz, the experiments require the simultaneous application of a conservative tracer to determine the travel-time distribution at the outlet of the column (fluorescein will be the obvious choice but other suitable tracers such as $\mathrm{NaCl}$ should also be feasible).

5. In order to obtain the best possible informative reactive transport parameters, we suggest using a travel-timebased modeling approach avoiding limitations of common advection-dispersion approaches for column experiments (Cortis et al., 2004) and therefore minimizing bias in the reactive transport parameters.

6. We include all relevant processes identified in the modeling of the laboratory experiments for the analysis of measured BTCs in the course of field tracer tests.

The so-derived data sets on sorption and decay parameters of Raz and Rru can be used to obtain relationships with possible environmental controls of their reactive transport, such as $\mathrm{pH}$, organic carbon content, or sediment texture.

\section{The Supplement related to this article is available online at doi:10.5194/hess-18-3151-2014-supplement.}

Acknowledgements. We are grateful to Herman Rügner and Peter Grathwohl for their helpful suggestions on the manuscript and furthermore thank Kevin Ferris for his advice on respiratory behavior of microorganisms. We furthermore thank Martin Gritsch, Julia Knapp and Stéphane Ngueleu Kamangou for their active assistance in the laboratory. This work was supported by grants from the Ministry of Science, Research and Arts of Baden-Württemberg (AZ $\mathrm{Zu} \mathrm{33-721.3-2),} \mathrm{the} \mathrm{Helmholtz} \mathrm{Centre} \mathrm{for} \mathrm{Environmental}$ Research, Leipzig (UFZ), the German Academic Exchange Service (DAAD), and the US National Science Foundation, grant \#EAR 08-38338.

Edited by: L. Pfister

\section{References}

Argerich, A., Marti, E., Sabater, F., Haggerty, R., and Ribot, M.: Influence of transient storage on stream nutrient uptake based on substrata manipulation, Aquat. Sci., 73, 365-376, doi:10.1007/s00027-011-0184-9, 2011.

Baker, M. D., Inniss, W. E., and Mayfield, C. I.: Effect of pH on the growth and activity of heterotrophic sediment microorganisms, Chemosphere, 11, 973-983, 1982.
Cartwright, C. D., Thompson, I. P., and Burns, R. G.: Degradation and impact of phthalate plasticizers on soil microbial communities, Environ. Toxicol. Chem., 19, 1253-1261, doi:10.1002/etc.5620190506, 2000.

Cirpka, O. A., Fienen, M. N., Hofer, M., Hoehn, E., Tessarini, A., Kipfer, R., and Kitanidis, P. K.: Analyzing bank filtration by deconvoluting time series of electric conductivity, Ground Water, 45, 318-328, doi:10.1111/j.1745-6584.2006.00293.x, 2007.

Cortis, A., Chen, Y. J., Scher, H., and Berkowitz, B.: Quantitative characterization of pore-scale disorder effects on transport in "homogeneous" granular media, Phys. Rev. E, 70, 041108, doi:10.1103/PhysRevE.70.041108, 2004.

De Hoog, F. R., Knight, J. H., and Stokes, A. N.: An improved method for numerical inversion of Laplace transforms, SIAM J. Sci. Stat. Comp., 3, 357-366, doi:10.1137/0903022, 1982.

Erban, T. and Hubert, J.: Determination of $\mathrm{pH}$ in regions of the midguts of acaridid mites, J. Insect Sci., 10, 1-12, doi:10.1673/031.010.4201, 2010.

Ettwig, K. F., Butler, M. K., Le Paslier, D., Pelletier, E., Mangenot, S., Kuypers, M. M. M., Schreiber, F., Dutilh, B. E., Zedelius, J., de Beer, D., Gloerich, J., Wessels, H. J. C. T., van Alen, T., Luesken, F., Wu, M. L., van de Pas-Schoonen, K. T., den Camp, H. J. M. O., Janssen-Megens, E. M., Francoijs, K. J., Stunnenberg, H., Weissenbach, J., Jetten, M. S. M., and Strous, M.: Nitrite-driven anaerobic methane oxidation by oxygenic bacteria, Nature, 464, 543-548, doi:10.1038/Nature08883, 2010.

Gelman, A. and Rubin, D. B.: Inference from iterative simulation using multiple sequences, Stat. Sci., 7, 457-472, 1992.

González-Pinzón, R., Haggerty, R., and Myrold, D. D.: Measuring aerobic respiration in stream ecosystems using the resazurinresorufin system, J. Geophys. Res.-Biogeo., 117, G00n06, doi:10.1029/2012jg001965, 2012.

González-Pinzón, R., Haggerty, R., and Argerich, A.: Quantifying spatial differences in metabolism in headwater streams, Freshwater Sci., 33, 798-811, doi:10.1086/677555, 2014.

Grathwohl, P.: Diffusion in Natural Porous Media - Contaminant Transport, Sorption/Desporption and Dissolution Kinetics, in: Topics in Environmental Fluid Mechanics Series, edited by: Chatwin, P., Dagan, G., List, J., Mei, C., and Savage, S., Kluwer Academic Publishers, Boston, 1998.

Grolimund, D., Borkovec, M., Federer, P., and Sticher, H.: Measurement of sorption isotherms with flow-through reactors, Environ. Sci. Technol., 29, 2317-2321, doi:10.1021/Es00009a025, 1995.

Haggerty, R., Argerich, A., and Marti, E.: Development of a "smart" tracer for the assessment of microbiological activity and sediment-water interaction in natural waters: the resazurin-resorufin system, Water Resour. Res., 44, W00d01, doi:10.1029/2007wr006670, 2008.

Haggerty, R., Marti, E., Argerich, A., von Schiller, D., and Grimm, N. B.: Resazurin as a "smart" tracer for quantifying metabolically active transient storage in stream ecosystems, J. Geophys. Res.-Biogeo., 114, G03014, doi:10.1029/2008jg000942, 2009.

Herbert, R. B., Malmstrom, M., Ebena, G., Salmon, U., Ferrow, E., and Fuchs, M.: Quantification of abiotic reaction rates in mine tailings: evaluation of treatment methods for eliminating ironand sulfur-oxidizing bacteria, Environ. Sci. Technol., 39, 770777, doi:10.1021/Es0400537, 2005. 
Hingston, F. J., Posner, A. M., and Quirk, J. P.: Anion adsorption by goethite and gibbsite I. The role of the proton in determining adsorption in envelopes, J. Soil Sci., 23, 177-192, doi:10.1111/j.1365-2389.1972.tb01652.x, 1972.

Kangasniemi, K. H.: Fluorescent Diagnostics for Imaging Dissolved Oxygen, Ph.D., University of Texas, Dallas, 131 pp., 2004.

Kasnavia, T., Vu, D., and Sabatini, D. A.: Fluorescent dye and media properties affecting sorption and tracer selection, Ground Water, 37, 376-381, doi:10.1111/j.1745-6584.1999.tb01114.x, 1999.

Lemke, D., Liao, Z., Wöhling, T., Osenbrück, K., and Cirpka, O. A.: Concurrent conservative and reactive tracer tests in a stream undergoing hyporheic exchange, Water Resour. Res., 49, 30243037, doi:10.1002/wrcr.20277, 2013a.

Lemke, D., Schnegg, P. A., Schwientek, M., Osenbrueck, K., and Cirpka, O. A.: On-line fluorometry of multiple reactive and conservative tracers in streams, Environ. Earth Sci., 69, 349-358, doi:10.1007/s12665-013-2305-3, 2013b.

Liao, Z., Lemke, D., Osenbrück, K., and Cirpka, O. A.: Modeling and inverting reactive stream tracers undergoing two-site sorption and decay in the hyporheic zone, Water Resour. Res., 49, 3406-3422, doi:10.1002/wrcr.20276, 2013.

McKinley, V. L. and Vestal, J. R.: Effects of acid on plant litter decomposition in an arctic lake, Appl. Environ. Microb., 43, 11881195, 1982.

Miller, C. T. and Weber, W. J.: Modeling the sorption of hydrophobic contaminants by aquifer materials, 2. Column reactor systems, Water Res., 22, 465-474, doi:10.1016/00431354(88)90041-3, 1988.

Östlund, P., Carman, R., and Edvardsson, U. G.: Sterilization of sediments by ionizing radiation, Appl. Geochem., 4, 99-103, doi:10.1016/0883-2927(89)90062-0, 1989.

Piwoni, M. D. and Keeley, J. W.: Basic concepts of contaminant sorption at hazardous waste sites, EPA Ground Water Issue, EPA/540/4-90/053, US Environmental Protection Agency, Robert S. Kerr Environmental Research Laboratory, Ada, OK, 1990.
Schoups, G. and Vrugt, J. A.: A formal likelihood function for parameter and predictive inference of hydrologic models with correlated, heteroscedastic, and non-Gaussian errors, Water Resour. Res., 46, W10531, doi:10.1029/2009wr008933, 2010.

Smith, S. A. and Pretorius, W. A.: The conservative behaviour of fluorescein, Water SA-Pretoria, 28, 403-406, 2002.

Stanaway, D., Haggerty, R., Benner, S., Flores, A., and Feris, K.: Persistent metal contamination limits lotic ecosystem heterotrophic metabolism after more than 100 years of exposure: a novel application of the resazurin resorufin smart tracer, Environ. Sci. Technol., 46, 9862-9871, doi:10.1021/Es3015666, 2012.

ter Braak, C. J. F. and Vrugt, J. A.: Differential evolution Markov chain with snooker updater and fewer chains, Stat. Comput., 18, 435-446, doi:10.1007/s11222-008-9104-9, 2008.

Wang, A. S., Angle, J. S., Chaney, R. L., Delorme, T. A., and McIntosh, M.: Changes in soil biological activities under reduced soil $\mathrm{pH}$ during Thlaspi caerulescens phytoextraction, Soil Biol. Biochem., 38, 1451-1461, doi:10.1016/j.soilbio.2005.11.001, 2006.

Weber, W. J., Mcginley, P. M., and Katz, L. E.: Sorption phenomena in subsurface systems - concepts, models and effects on contaminant fate and transport, Water Res., 25, 499-528, doi:10.1016/0043-1354(91)90125-A, 1991.

Wöhling, T. and Vrugt, J. A.: Multiresponse multilayer vadose zone model calibration using Markov chain Monte Carlo simulation and field water retention data, Water Resour. Res., 47, W04510, doi:10.1029/2010wr009265, 2011.

Wöhling, T., Bidwell, V. J., and Barkle, G. F.: Dual-tracer, nonequilibrium mixing cell modelling and uncertainty analysis for unsaturated bromide and chloride transport, J. Contam. Hydrol., 140, 150-163, doi:10.1016/j.jconhyd.2012.08.001, 2012. 